\title{
La opinión en la prensa española: marcos interpretativos del comunicado de la OMS sobre la carne
}

institucional.us.es/ambitos/

\section{José Ignacio Armentia Vizuete}

Universidad del País Vasco

ignacio.armentia@ehu.eus

\section{Flora Marín Murillo}

Universidad del País Vasco

flora.marin@ehu.eus

\section{María Elena Olabarri Fernández \\ Universidad del País Vasco \\ mariaelena.olabarri@ehu.eus}

English Version: The view in the Spanish press: interpretive frameworks WHO statement on meat.

\section{Resumen}

El 26 de octubre de 2015, la Organización Mundial de la Salud difundió un comunicado en el que informaba de la carcinogenicidad de la carne roja y procesada. En este artículo se estudia cuál fue el tratamiento que El País, El Mundo, La Vanguardia y El Correo dieron a este tema en sus páginas de opinión. Desde la teoría del framing y previo análisis del discurso se examinan los recursos estilísticos, argumentos, autoría, fuentes y funciones del encuadre publicados en estos medios. Los resultados obtenidos permiten hablar de un marco crítico mayoritario que utilizó argumentos desdramatizadores para atenuar y minimizar la percepción del riesgo

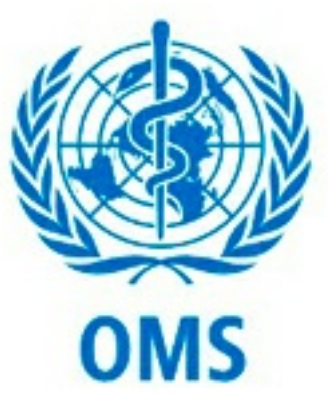
inicial.

\section{Palabras clave}

Riesgo, comunicación, framing, opinión, prensa.

Abstract

On 26 October 2015, the World Health Organization released a statement reporting the carcinogenicity of red and processed meat. This article examines what treatment was given to this issue by newspapers such as El

Pais, El Mundo, La Vanguardia and El Correo in its opinion pages. Style resources, arguments, authorship, sources and functions of published frameworks in the media mentioned are analyzed from the theory of framing, after a discourse analysis. The results achieved allow us to speak of a main critical framework that used different arguments to mitigate and minimize the initial risk perception.

Keywords Risk, comunicación, framing, opinión, prensa.

\section{INTRODUCCIÓN}

La Organización Mundial de la Salud (OMS) difunde el 26 de octubre de 2015 un comunicado donde hace públicos los resultados obtenidos por el Centro Internacional de Investigaciones sobre Cáncer (IARC, por sus siglas en inglés) acerca de la carcinogenicidad del consumo de carne roja y de carne procesada. Tras revisar de forma exhaustiva la literatura científica acumulada, 22 expertos de 10 países clasificaron el consuno de carne roja "como probablemente carcinógeno para los humanos" y la carne procesada como "carcinógena para los humanos" 
(http://www.who.int/mediacentre/news/releases/2015/cancer-red-meat/es/).

El 27 de octubre todos los medios de comunicación se hacen eco de esta noticia y la prensa española lleva el tema a sus portadas. Aunque existía el consenso generalizado de estar ante una información ya conocida, la alarma no se hizo esperar.

El 29 de octubre, la OMS sale al paso ante las muestras de inquietud y consultas recibidas con una nueva declaración. En ella confirma que el estudio no hace sino reiterar las recomendaciones de otro trabajo anterior realizado en el 2002, y matiza "que no se pide a la población que deje de comer carnes procesadas, sino que se indica que la reducción del consumo de estos productos puede disminuir el riesgo de cáncer colorectal" (http://www.who.int/mediacentre/news/releases/2015/cancer-red-meat/es/).

El riesgo al que hacía referencia no es eminente y pone en evidencia la complejidad del propio concepto y sus distintas tipologías, ya que pese a su aparente simplicidad sus definiciones varían sustancialmente dependiendo del ámbito de estudio, el riesgo evaluado e incluso el propio evaluador. Así, Jaeger et al. (2001) definen el riesgo como una situación o acontecimiento en el cual algo del valor humano (incluyendo a los humanos mismos) se ha puesto en juego y el resultado es incierto.

El riesgo implica la incertidumbre de que algo malo suceda y ponga en peligro el bienestar o la vida de las personas afectadas. El hecho de que sea difícil de predecir hace que algunos autores distingan entre riesgos normales y riesgos excepcionales o catastróficos (López y Luján, 2000). Los primeros conllevan que el peligro pueda ser calculado de manera objetiva o al menos estimarse; los segundos son extremadamente imprevisibles y de ahí que sea imposible su prevención.

Pese a estos esfuerzos de control y prevención no hay que olvidar que la incertidumbre manifiesta convierte al riesgo en algo intangible y etéreo, dando lugar a una visión del riesgo constructivista, donde las estructuras sociales y la cultura desempeñan un papel fundamental para su percepción. En este sentido, el filósofo alemán, Beck, reconoce que la intensificación de los riesgos en la sociedad actual no es ajena a nuestra visión sobre ellos, de tal manera que ambos aspectos convergen y se fortalecen mutuamente "porque los riesgos son riesgos en el conocimiento, los riesgos y su percepción no son dos cosas diferentes sino una y una misma cosa."(Beck, 2002: 62).

La construcción social del riesgo ligada a esta perspectiva puede desembocar como apunta Farré en posturas extremas, "en una especie de relativismo que prácticamente hace desaparecer el riesgo que solamente se manifiesta a través de sus percepciones" (Farré, 2005: 103). De esta manera asumir que el riesgo sólo existe si lo percibimos como tal, sería igual a equiparar el riesgo real con el riesgo percibido, entendiendo que uno no existe sin el otro, y "olvidando que la definición del riesgo tiene lugar en el contexto social donde se acuerdan las bases para su domesticación.” (Farré, 2005: 104).

Es precisamente en ese contexto al que hacía alusión Farré donde el papel de los medios de comunicación es fundamental. La comunicación de riesgos, atañe tanto a las instituciones y particulares que los detectan y comunican en primera instancia como a los medios de comunicación que median entre estos entes y la ciudadanía, haciendo del riesgo algo real y tangible, amplificando o contrarrestando sus efectos más inmediatos. Algunos autores como Saura (2005), Löfstedt (2010), Gonzálo y Farré (2011), y Rutsaert y Regan (2013), entre otros, se han centrado tanto en la comunicación de riesgos a nivel teórico como en la responsabilidad de los medios en la percepción de la seguridad alimentaria.

Para Gil Calvo "son los medios los que ahora definen la realidad de los hechos, decidiendo qué riesgo es real y cuál no. Pero también son los medios los que califican los riesgos evaluando su nivel de peligrosidad para dictaminar cuáles son normales, cuáles relegan a la crónica de sucesos y cuáles son catastróficos, a los que magnifican después de elevarlos a la categoría de acontecimientos mediáticos" (2006: 111).

En este estudio se ha seleccionado el género de opinión porque a diferencia de los géneros informativos o interpretativos, en estos la actitud del periodista asume como esencial el enjuiciamiento y valoración de los hechos más que los hechos en sí mismos. "Los géneros de opinión argumentaran partiendo del discurso que genera la búsqueda de las causas y el análisis de sus efectos para juzgar actos y realidades" (Santamaría y 
Casals, 2000: 183).

Los editoriales como carta de presentación del periódico reflejan su punto de vista, explican, interpretan, valoran, emiten juicios, sitúan los antecedentes, evalúan las causas e incluso emiten pronósticos o sugieren soluciones. Sin editoriales, como muy bien dice Mostaza (1971), "el periódico quedaría reducido a mera superficie; no sería el cuerpo de la actualidad, sería su espectro" (Mostaza,1971: 171).

Del mismo modo, la columna "es un artículo de opinión que puede ser razonador o lo contrario, falaz, orientador o enigmático; analítico o pasional; enjuiciativo o narrativo; y siempre valorativo, subjetivo, porque no puede ser de otro modo" (Santamaría y Casals, 2000: 288).

El componente valorativo e interpretativo del género permite observar la riqueza de los recursos empleados y las estrategias interpretativas de los diarios a la hora de enmarcar los acontecimientos.

Las cartas al director no son ajenas a este cometido. Estas revisten especial interés porque al margen de la criba que realizan los editorialistas ligada a aspectos formales, extensión, corrección, estilo, es un apartado "que, si se sabe manejar con habilidad, se convierte en un arma eficaz al servicio de la línea editorial del medio, un recurso más para modelar la opinión pública" (Abril, 1999: 161).

Los ciudadanos acuden a los medios en busca de respuestas. Estos, en ocasiones se convierten en altavoces de intereses contrapuestos, o en voceros de expertos, minimizan los riesgos o amplifican las alarmas y su trabajo repercute de manera directa en la percepción que el público tiene del peligro. El miedo o la indiferencia que puedan llegar a generar dependerá del enfoque particular que den a la información y por tanto su grado de responsabilidad merece toda nuestra atención.

\section{OBJETIVOS E HIPÓTESIS}

Si como dice Sádaba "se utilizan los frames como marcos estructurados capaces de cambiar el rumbo de las percepciones sociales” (Sádaba, 2007: 210), lo que esta en juego en este análisis es conocer cómo la prensa utilizó los recursos y estrategias para cambiar la percepción social del riesgo. Para ello se han planteado los siguientes objetivos:

1. Describir e interpretar los discursos que sobre el comunicado de la OMS se vertieron en los textos de opinión.

2. Reconocer y sistematizar los dispositivos y argumentos utilizados para enmarcar el comunicado.

3. Establecer cuáles fueron los marcos interpretativos prioritarios y las estrategias de encuadre en cada caso.

4. Comparar y matizar los diferentes marcos interpretativos y la relevancia otorgada en cada medio a los dispositivos y argumentos.

5. Indagar si hubo o no coincidencias entre la línea editorial de cada medio y la opinión expresada por sus articulistas, columnistas y lectores.

Las hipótesis de las que parte este análisis y que intentan ser contrastadas a lo largo de este estudio son las siguientes:

H.1. El contenido del comunicado de la OMS no estuvo exento de polémica y su relevancia fue constatable a través de su presencia en las páginas de opinión durante un tiempo prolongado.

H.2. Los marcos interpretativos dominantes fueron construidos a través de paquetes de argumentos y dispositivos de encuadre.

H.3. Los argumentos mayoritarios estuvieron encaminados a mitigar la alerta y atenuar sus consecuencias económicas. 
H.4. Durante el mes estudiado los medios contribuyeron a cambiar la percepción social del riesgo presente en el contenido original de la OMS.

H.5. No hubo homogeneidad en la cobertura del comunicado de la OMS por parte de todo los periódicos.

H.6. Los intereses de los sectores ganaderos y la industria agroalimentaria condicionaron los encuadres seleccionados por algunos diarios.

\section{METODOLOGÍA}

Para Rabadán "el análisis de la estrategia de encuadre requiere una revisión de una muestra exhaustiva que abarque un periodo extenso de tiempo para poder detectar tendencias estructurales consolidadas" (López Rabadán, 2010: 246). En este sentido, el comunicado de la OMS al convertirse en una noticia de actualidad prolongada se presta a este modelo de análisis.

Para alcanzar los objetivos propuestos se han recopilado todos aquellos editoriales, columnas, artículos de opinión y cartas al director publicados desde el 27 de octubre 2015 hasta el 30 de noviembre del mismo año.

Los periódicos elegidos para esta muestra han sido El País, El Mundo, La Vanguardia y El Correo. Los tres primeros fueron la cabeceras de información general con mayor circulación en 2015, según los datos de introlOJD. El Correo ocupa la sexta posición en dicho ranking, pero es el principal medio español de información regional y local, además de pertenecer al Grupo Vocento, en el que también se incluye $A b c$, cuarto diario con mayores ventas. Junto a la alta difusión de los rotativos elegidos, se ha buscado que existiese un equilibrio en la muestra entre la prensa de ámbito estatal, editada en Madrid, y la de la periferia.

\begin{tabular}{lc} 
Tabla 1. Difusión de los medios seleccionados en 2015 \\
\hline Diario & Difusión \\
\hline EI País & 221.390 \\
La Vanguardia & 129.073 \\
EI Mundo & 126.369 \\
El Correo & 72.956 \\
\hline
\end{tabular}

Fuente: OJD

En este modelo de estudio se han tenido en cuenta planteamientos metodológicos como el de Alcíbar Cuello (2015) que apuesta por compaginar el análisis del discurso y la teoría del framing, como propuesta pragmáticodiscursiva para analizar artículos editoriales.

Así, las informaciones seleccionadas se han descrito e interpretado a través de un análisis del contenido, entendido este como "una técnica de investigación para formular inferencias identificando de manera sistemática y objetiva ciertas características específicas dentro de un texto" (Holsti, 1969: 5). De ahí que los recursos estilísticos y el tono empleado sean también reseñados, constituyendo un primer acercamiento al contenido explícito y subyacente de los textos. Asimismo, las citas directas sirven para ilustrar los diferentes sesgos y estrategias persuasivas, ya que como dice Van Dijck "en lugar de trasmitir las creencias dominantes directamente, los medios de comunicación construyen una estructura interpretativa” (1997: 70).

Este concepto de estructura interpretativa entronca con la idea del framing que en los medios de comunicación responde a un argumento central que dota de sentido a la información. Se trata por tanto de hallar en un segundo estadio los diferentes frames o marcos interpretativos a través de la sistematización de los recursos y dispositivos utilizados, lo que Van Gorp (2007) denomina frame package.

Si Entman (1993) definió las diferentes funciones del encuadre como "los ideales componentes con que debe contar según el normativismo de los cursos de redacción periodística, toda noticia «bien construida»" (Cuvardic, 
2002: 87), en este caso se ha intentado adaptar esas funciones al género de opinión. Para este cometido se ha tenido en cuenta que la estructura del editorial puede cambiar ligeramente de un medio a otro pero, por lo general, englobará, la exposición del tema con sus antecedentes y causas, si las hubiera, las consecuencias que se derivan y sus posibles soluciones o recomendaciones. En el caso de las columnas y artículos de opinión, aun con un estilo más libre, en ocasiones literario, y con una estructura menos encorsetada, mantienen muchas de estas disposiciones. De hecho, Santamaría y Casals reconocen entre los argumentos analíticos que "más se utilizan en los artículos de opinión de los periódicos: las causas y las consecuencias" (2000: 177).

De ahí, que se tengan en cuenta las 4 funciones clásicas del encuadre de Entman (1993:52); La tipología de base temporal de Eilders y Lüter (2009) y los cinco grandes encuadres genéricos diseñados por Semetko y Valkenburg (2000).

De las aportaciones anteriores y lectura crítica de los artículos se han inducido cinco funciones de encuadre que pueden esclarecer la estructura de los distintos marcos interpretativos:

\section{Gráfico 1. Funciones de encuadre/OMS}

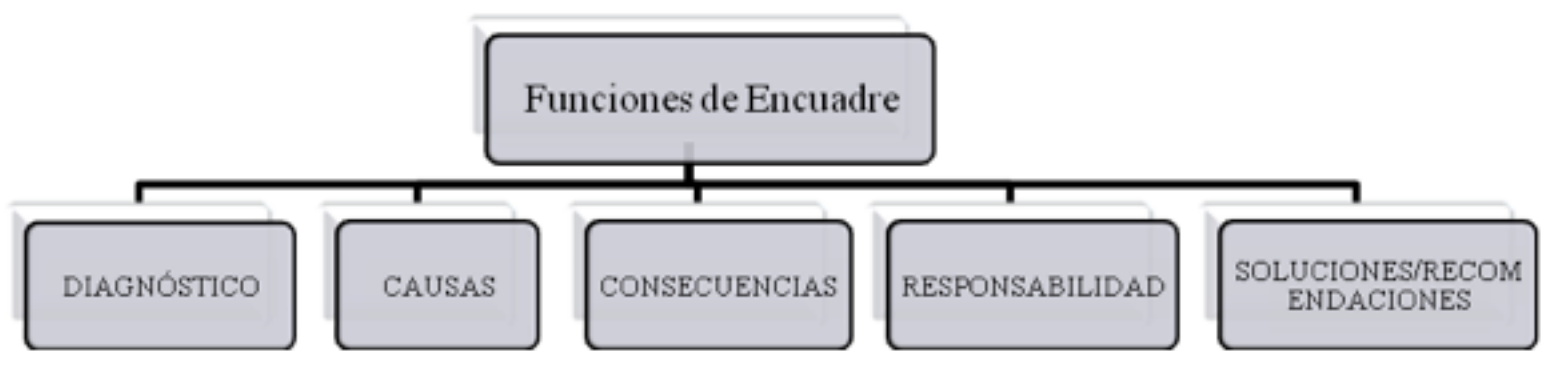

\section{Fuente: Elaboración propia}

La Real Academia de la lengua define diagnóstico, con una doble acepción: "Recoger y analizar datos para evaluar problemas de diversa naturaleza" y "Determinar el carácter de una enfermedad mediante un examen de sus signos". En el contexto analizado el diagnóstico se puede aplicar tanto al contenido del comunicado presentado por la OMS, como a la posterior evaluación y juicio que sobre el mismo hicieron los medios. (http://dle.rae.es/)

En este dispositivo se tienen en cuenta los juicios de valor que se emitieron sobre el propio comunicado, los antecedentes y la contextualización a la que dio lugar. Las causas en la tipología de Eilders y Luters (2009) se engloban en el diagnóstico, sin embargo aquí se ha decidido deslindarla del mismo, ya que en el análisis crítico y exhaustivo que se le presupone a un texto de opinión estas deberían estar siempre presentes, por lo que su ausencia también resulta reveladora.

Semetko y Valkjemburm (2000) destacan las consecuencias económicas que en esta investigación también tienen un peso importante, pero no se pueden dejar de lado otras consecuencias de tipo políttico, sanitario, etc.

Tanto Entman (1993) como Semetko (2000) recogen el concepto de responsabilidad, tan importante en un texto de opinión, que trata de enjuiciar un problema y señalar a los responsables. Quiénes sean estos en cada caso será determinante para configurar un marco interpretativo $u$ otro.

Y por último habría que referirse a las soluciones y recomendaciones, a menudo el colofón de muchos de los artículos, columnas y editoriales.

Una vez definidas las funciones de encuadre que responden a grandes rasgos a la estructura de los contenidos 
analizados, se han extraído los recursos lógicos o de razonamiento (reasoning devices) que no son sino aquellos argumentos que justifican la credibilidad del encuadre, y representan el armazón que lo sostiene (Pan y Kosicki, 1993; Van Gorp, 2005).

No hay que olvidar que el género de opinión se caracteriza por su hacer persuasivo y en él la lógica discursiva y la retórica cobran gran importancia. "En este sentido el discurso argumentativo apunta, si no siempre a convencer, al menos sí a establecer la justeza de una actitud, de un razonamiento o de una conclusión" (Gaitán y Piñuel, 1998: 296)

La presencia o no de los dispositivos enunciados, el uso de unos argumentos u otros, serán aspectos esenciales para definir los marcos interpretativos. Como decía Entman: "Most frames are defined by what they omit as well as include, and the omissions of potential problem definitions, explanations, evaluations, and recommendations may be as critical as the inclusions in guiding the audience." (Entman,1993: 54).

La teoría del framing y el análisis de contenido aplicado al tratamiento que la prensa dispensa a los temas de salud ha dado lugar a numerosas y fructíferas investigaciones. Cabe destacar el estudio de Enguix y Gallardo (2014) sobre los encuadres informativos en El País, El Mundo y La Vanguardia, del Daño Cerebral Adquirido (DCA) y más recientemente, el texto de Atanasova y Koteyko (2016) sobre la prominencia que de los diferentes encuadres sobre la obesidad se realizan en la prensa on line alemana y británica.

\section{ANÁLISIS CRÍTICO}

Desde el 27 de octubre hasta el 30 de noviembre de 2015, en los cuatro diarios analizados se publican un total de 30 piezas, entre editoriales, cartas al director, artículos de opinión y columnas.

La distribución de estos contenidos se puede observar en la tabla 2.

Tabla 2. Contenidos de opinión publicados

\begin{tabular}{lcccc} 
Periódicos & Editoriales & Art/columnas & Cartas al director & Total \\
\hline El Correo & 1 & 3 & 1 & 5 \\
El País & 2 & 4 & 2 & 8 \\
La Vanguardia & 2 & 6 & 2 & 10 \\
El Mundo & 1 & 5 & 1 & 7 \\
Total & 6 & 18 & 6 & 30 \\
\hline
\end{tabular}

Fuente: Elaboración propia

El hecho de que todos los diarios publicasen al menos un editorial sobre este tema y dos de ellos, El País y La Vanguardia, le dedicasen hasta dos editoriales da idea de la relevancia que cobró el comunicado de la OMS en la prensa española.

El número de artículos y columnas de opinión también es significativo y aunque se muestra en términos absolutos, destacando La Vanguardia con 6 piezas, se mantiene el equilibrio porcentualmente respecto a la totalidad de contenidos publicados en cada medio.

\subsection{Editoriales}

El Correo es el primero en enjuiciar el contenido emitido por la OMS, el 27 de octubre. La Vanguardia lo hará un día después, y El País y El Mundo, dejarán reposar la información para emitir su valoración tres días más tarde, el 29 .

Quizás sea esta premura por parte de El Correo lo que hace que su enfoque sea ligeramente distinto al del resto de los medios analizados. El argumento que apela al equilibrio y la moderación está presente en el subtítulo: "La salud pública y el bienestar personal no están reñidos a la hora de comer, pero demandan 
contención y equilibrio". Desde el inicio se decanta por un enfoque explícitamente positivo y legitimador respecto a todo lo expresado por la OMS. Aun adelantando, como es la tónica general, que estamos ante una información de sobra conocida, se expresa en términos elogiosos reconociendo que "concede rango institucional a una advertencia que desde hace dos décadas forma parte del consenso en la oncología médica".

El mensaje adquiere para el medio rango universal y lejos de cuestionarse la validez del mismo se define como un dictamen "inapelable en cuanto a los riesgos que comporta el abuso en el consumo habitual de tales alimentos".

Este editorial estaría a medio camino entre el modelo "explicativo" y el "admonitorio" (Santamaría y Casals, 2000: 275-276) "pues explica la naturaleza del acontecimiento y su trascendencia social " y advirtiendo de los peligros "apela al lector a preocuparse por los problemas tratados".

La admonición se centra en señalar las dudas sobre el papel que el poder ejecutivo y judicial deberán desempeñar en este contexto.

Los tres diarios restantes utilizaran fundamentalmente argumentos de equilibrio y moderación a la hora de exponer los hechos.

Así, El Mundo, el 29.10.2015, tras advertir de que "nadie puede dudar de las conclusiones de la OMS", atempera el estudio a través de su interpretación: "el informe sobre las carnes procesadas viene a decir que seguir una dieta equilibrada es lo mejor para evitar posibles complicaciones posteriores".

El País hace lo propio y el mismo día 29 de octubre en su primer editorial deja claro que "el trabajo no plantea objeciones de rigor, ni oportunidad". Al final recomienda al consumidor que esté bien informado y el equilibrio en sus hábitos alimenticios: "lo apropiado es que tomen las cantidades de carne roja que las autoridades sanitarias recomiendan para una dieta saludable".

La Vanguardia el 28 de octubre titula con un escueto pero contundente "Carne y Cáncer" su primer editorial y, después de dar el visto bueno a la divulgación del informe por parte de la OMS, contextualiza los datos, los interpreta y reconduce para ofrecer un diagnóstico más crítico. Enfatiza el "probable" del comunicado, la "cautela" con la que hay que afrontar su lectura y termina recomendando moderación: "...la necesidad de seguir una alimentación variada y equilibrada en línea con la dieta mediterránea que impera en España”. Este es el diario en el que los argumentos desdramatizadores tienen mayor peso. De hecho, la mayoría de sus estrategias discursivas están encaminadas a bajar el tono de la alarma y cuestionar la sensación de riesgo generada. Así, se apela a un argumento de autoridad, mencionando una imprecisa "mayoría" de sociedades médicas y científicas que refrendan la moderación como respuesta al mismo. Incluso, aunque de forma tangencial, se hace referencia al argumento "Beneficios de la carne" para señalar "su importante valor nutricional".

Otro de los argumentos que justifican a su vez el diagnóstico en clave crítica, redefiniendo la alarma como injustificada, serán los fallos en la comunicación de riesgos, que se atribuyen prioritariamente a la propia OMS, y en menor medida a los mismos medios de comunicación. Así, sobre todo El Mundo y El País, hacen responsable a la OMS de provocar alarmismo, al no tener en cuenta que se dirigen a un público no experto, no utilizar un estilo divulgativo, existir un déficit informativo y no presentar recomendaciones específicas. Una vez expuestos los fallos, las propuestas de los diarios intentan subsanarlos: “..hay que exigir a esa organización y a los organismos nacionales que difunden esos informes una imprescindible labor divulgativa”. (El Mundo, 29.10.2015); "Debería revisar sus métodos" (El País, 29.10.20159;" adolece de la ausencia de recomendaciones específicas lo que ha contribuido a amplificar el alarmismo" (La Vanguardia, 28.10.2015).

La Vanguardia y El País, volverán a editorializar días después, el 1 de noviembre y en esta ocasión aprovecharán para ahondar en las causas y las consecuencias del informe de la OMS.

La Vanguardia, con un elocuente titular "Golpe al sector cárnico", y desde un enfoque economicista que no trata de ocultar reflexiona sobre las consecuencias del comunicado en un sector como el cárnico de gran importancia en el Estado y también en Cataluña y cuyas "cuantiosas inversiones" se ven amenazadas. Las consecuencias económicas priman sobre las sanitarias, que son deliberadamente apartadas: "al margen de la 
cuestión sanitaria".

El País, por su parte, incide directamente en las causas que provocan la carcinogenicidad de la carne y ya desde el editorial deja claras sus intenciones: "la carne no mata, los añadidos sí". El uso de anabolizantes, esteroides, y otros productos en la alimentación de los animales, así como los conservantes, antioxidantes, y otros aditivos que se utilizan para las carnes tratadas serían los verdaderos causantes del riesgo de cáncer. Por tanto la industria alimentaria sería la responsable y las medidas a tomar estarían dirigidas a la inversión en investigación, ya que "tiene que ofrecer productos que no dañen la salud, ni por la materia prima ni por sus añadidos".

La Vanguardia aunque coincide en el diagnóstico se centra en la victimización de la industria cárnica, y aun reconociendo su responsabilidad, la comparte con las autoridades sanitarias, a las que hace responsables en última instancia de tomar las medidas necesarias, estableciendo nuevas reglamentaciones si fuesen necesarias, pero dejando constancia de que estas son "ya muy elevadas" para el sector cárnico.

Se puede concluir, que El Correo es el diario que más claramente se decanta a favor del comunicado y su utilidad, con un enfoque positivo. El País, en su segundo editorial también se posiciona a favor del mismo, siendo el único que ahonda en las causas y responsabiliza a las industrias cárnicas, estableciendo recomendaciones encaminadas a subsanar sus riesgos. La Vanguardia se inclina en su segundo editorial por destacar las consecuencias en forma de pérdidas económicas, siendo el diario más proclive al sector y la industria cárnica.

El resto de los periódicos y editoriales utilizan para su diagnóstico argumentos claramente desdramatizadores, optando por contextualizar los efectos de la ingesta de carne, y la crítica explícita a los fallos en la comunicación de riesgo.

\subsection{Artículos/columnas de opinión}

Entre los cuatro diarios analizados se publican un total de 18 contenidos, entre artículos y columnas de opinión. Los autores de los mismos, son mayoritariamente los colaboradores habituales, Quim Monzó, Manuel Alcántara o Magda Carlas. Sólo Quim Monzó recurre a este tema en cuatro ocasiones, aunque en dos de ellas su aparición sea anecdótica.

En El País y El Correo firman dos personas expertas en el ámbito de la salud, Javier Padilla, médico y master en economía de la salud, (El Correo) y Miguel Porta, catedrático de Salud Pública en la Universidad Autónoma de Barcelona (El País). El Mundo contará con la presencia de Pedro Nuño De La Rosa, crítico gastronómico y La Vanguardia con su colaboradora habitual y nutricionista, Magda Carlas. Solo este último diario publicara un artículo de opinión de alguien cercano a la industria alimentaria, como es el caso de Josep Lagares, Presidente Ejecutivo de Metalquimia, empresa española dedicada al estudio de la Ciencia y la Tecnología de la carne.

El Correo publica dos columnas de opinión y un artículo. El día 28 de octubre, Manuel Alcántara, juega con la polisemia del término "régimen" para partiendo del anuncio de la OMS, terminar hablando de regímenes políticos, y en concreto de la hipotética instauración de la República catalana. A pesar de su carácter anecdótico, el autor despliega todo un repertorio de refranes y frases hechas para con humor y cierta dosis de ironía suavizar lo anunciado por la OMS. El efecto desdramatizador es evidente: "Todos sospechosos, hasta los chuletones del benemérito cerdo y el jamón, que es el mejor amigo del hombre, en competencia con el perro y además, no muerde a los extraños". Dos días después, y casi como contrarréplica a la columna anterior José María Romera, edita "Razones cárnicas", donde desmonta los mensajes de "esos apóstoles del solomillo", que refugiándose en el humor han intentado deslegitimar los dictámenes de la OMS. Para el autor la "certeza objetiva", "el método científico" las "razones bioquímicas" no pueden ser rechazadas por el carnicero que protege su negocio, o el temerario "de algo hay que morir". El uso de la ironía es el fundamento de esta argumentación por el ridículo que en cierta medida recurre también al ad hominen. Aunque el diagnóstico es favorable al final en la recomendación reitera el manido argumento de moderación, intentando así alcanzar el equilibrio: "..habrá que ir pensando en alejarse prudentemente de las carnes por la cuenta que nos trae". 
Por último, y desde la autoridad que confiere ser experto en economía de la salud, Javier Padilla desgrana en su artículo "De carnes, cánceres y el papel de los Estados" (03.11.205) toda una serie de argumentos que suavizan el contenido del comunicado restándole gravedad. Así, alude de forma didáctica a los fallos en la comunicación, que dieron lugar a "reacciones desproporcionadas y afirmaciones tan erróneas como alarmistas". Algunos de estos errores en la comunicación de riesgos fueron según Padilla "omitir cifras concretas o, de señalarlas, hacerlo siempre con datos de riesgo relativo y no con riesgos absolutos". Una vez aclarado el verdadero alcance del comunicado y sin entrar en las causas que inciden en la carcinogenicidad de la carne, las soluciones son de alcance institucional y no individual ya que se insta a los gobiernos para que adopten políticas de alimentación saludable. Estas políticas irían desde "favorecer fiscalmente la alimentación saludable" hasta la intervención en la industria alimentaria.

El Mundo, publica dos columnas y tres artículos de opinión. Al igual que en los editoriales, tanto en la columna firmada por Víctor de la Serna ("Periodismo, cáncer y alarmismo", 29.10.2015), como en el artículo de Mónica Nombella ("Dieta Mediterránea", 28.10.2015), el diagnóstico se dirige a atenuar la alarma creada. Esta última considera que "la OMS debería plantearse mejorar su sistema de comunicación, para no alarmar a la población con declaraciones vidriosas" y pone el acento en las recomendaciones ligadas al "Equilibrio": "Hay que comer de todo, disfrutando de los platos de la dieta mediterránea y tratando de no abusar de nada" .

Los "fallos en la comunicación" son también empleados por De la Serna, quien extiende los errores también a los medios de comunicación como responsables de la alarma injustificada: "Los periodistas iban desde la mala interpretación (...) a callarse las preguntas obvias". El autor emplea un argumento ejemplarizante para contrastar las buenas prácticas del The New York Times, frente a las malas prácticas tanto de la OMS como de los Medios de comunicación en general.

El día 30 de octubre Manuel Hidalgo en su artículo "la carne y la vida", intenta suavizar el contenido del comunicado recurriendo a los argumentos ad hominen, y teniendo como víctimas a los expertos a quienes intenta deslegitimar apelando a la "falta de credibilidad". Con grandes dosis de humor son descritos como personas incomodas, "te amargan la vida", de opiniones cambiantes y por tanto poco creíbles. Al final, y mediante un silogismo concluye: "Como estamos hechos de carne, estamos hechos de cáncer".

Con un estilo diferente, pero con argumentos que invocan también la "falta de credibilidad" el crítico en gastronomía Pedro Nuño de la Rosa en su artículo “¿Carne o pescado?”, parte de la premisa de que el ser humano es "carnívoro por naturaleza". Este hecho sería ignorado por los expertos, quienes son infravalorados describiéndolos como "gurús, curanderos, galenos, físicos". El escepticismo que estos sujetos despiertan en el autor está justificado dado que "se han venido equivocando muchas veces a lo largo de la Historia sobre lo conveniente al común de los humanos....".

Por si esto no fuera suficiente para cuestionar el informe de la OMS, utiliza el argumento de la "confrontación" refiriéndose a otro artículo publicado el día anterior donde se desmentían los peligros cárnicos-cancerígenos en la región.

El propio De la Rosa se expone a sí mismo como modelo ejemplar y confiesa que no dejará de degustar "un arroz con conejo y caracoles, unos contundentes callos o una exquisita pierna de cordero al horno".

La réplica a estos dos artículos y sus argumentos claramente desdramatizadores se publica el dos de noviembre bajo el título "chuletón de estupidez", firmado por Álvaro Colomer. En su artículo, al igual que hiciese José María Romera en El Correo, aporta argumentos para sostener la veracidad del comunicado y la necesidad de tomarse en serio sus dictámenes. Los merecedores de la crítica en esta ocasión son los ciudadanos por menospreciar a los expertos y tomárselo a la ligera. La recomendación final insta a los mismos para que "hagan caso a los expertos" y se vayan preparando.

El País publica tres artículos de opinión y una columna, y en al menos dos se alude a los "fallos en la comunicación". Uno de ellos, "La política, el placer y la carne" (27.10.2015) está firmado por un experto, Miguel Porta, Catedrático de Salud Pública en la Universidad Autónoma de Barcelona. Aunque en dicho texto el autor sostiene "el rigor científico" de la investigación, ofrece argumentos para un diagnóstico que minimiza la alarma inicial. Estos giran tanto en torno a los "beneficios de la carne", ya que no sólo se recuerdan sus virtudes 
nutritivas, sino también su "valor cultural y emocional"; como alrededor de errores comunicativos como "una información incompleta sobre lo que se puede o no comer" y la inconcreción sobre las medidas políticas o personales que se pueden llevar a acabo. Este último requerimiento es esgrimido como una necesidad para buscar el equilibrio entre la política, el placer y la carne, que dan título al artículo. Quizás, lo que más llame la atención es que Porta utilice como estrategia de persuasión su propia experiencia cotidiana a un nivel más emotivo que racional: "Cuántas parrilladas no habremos comido en familia y amistad".

Otro de los contenidos que alude a los fallos en comunicación es la columna de Julio Llamazares titulada "La carne" (02.11.2015). En esta ocasión el diagnóstico vuelve a ser lo infundado de la alarma, ya que, echando mano de la estrategia del absurdo y el uso retórico de la hipérbole, consigue sin decirlo explícitamente cuestionar la validez del comunicado. Llamazares afirma que según la OMS, la carne roja "es tan dañina como una bomba atómica", para más adelante advertir de que "la Organización Mundial de la Salud no ha dicho nada concreto y me temo que no lo vaya a hacer".

Somete al lector a la paradoja del absurdo haciéndonos saber que todo lo que comemos es cancerígeno y, por lo tanto, comer o no comer nos conducen al mismo final. “¿Cómo vamos a sobrevivir entonces?”, se pregunta el autor.

La misma estrategia del absurdo, con toques de humor, es empleada por Javier Sampedro, quién en su artículo, "Carne saludable y abundante sobre seis patas" (02.11.205), cuestiona la credibilidad de la OMS, oponiendo su informe a otro de la FAO (Organización para la Alimentación y la Agricultura) que propone como alternativa a la carne alimentarse de insectos.

Por último, Fernando Gualdoni, el domingo 1 de noviembre, en el artículo "Los Yanomamis no sufren de hipertensión" echa mano del comunicado de la OMS como pretexto para hablar de los abusos alimentarios y en concreto de la sal en los países desarrollados. Gualdoni utiliza una fuente experta como es Jared Diamond, biólogo y fisiólogo evolucionista estadounidense y autor del libro El mundo hasta ayer, para sostener un argumento centrado en el equilibrio y la moderación. Así, finaliza diciendo que Diamond "en ningún momento dice que no hay que tomar sal, sino hacerlo en su justa medida como en el caso de la carne roja".

En La Vanguardia, se publican un artículo de opinión y cinco columnas. En general, la mayoría de los columnistas abogan por una lectura más moderada y menos alarmista del comunicado. La OMS es desacreditada mediante todo tipo de argumentos. Francesc-Marc Álvaro, autor de "El rito de la OMS" (30.10.2015), sostiene que se trata de una nueva religión, y por tanto se debe a una serie de rituales para mantener a sus fieles. Se entiende que la metáfora aleja a esta organización de la racionalidad para adentrarse en el mundo de la fe: "La OMS es una nueva iglesia que tiene como dios la salud".

Josep Lagares, Presidente Ejecutivo de Metalquimia, el 1 de noviembre, recurre al lenguaje belicista para decir que nos encontramos "en los inicios de una guerra mundial contra la proteína" de la que la OMS sería su abanderada.

Otro argumento para restar credibilidad a la OMS, será confrontar su informe con otros estudios. Así, Quim Monzó alude en, "La tarará sí, la tarara no" (31.10.2015), a un estudio de la unidad de Biotecnología y Terapia Experimental del Instituto Universitario de Oncología del principado de Asturias "que demuestra que el consumo de ciertos tipos de jamón y de chorizo contribuyen a reducir la aparición del cáncer colorrectal". Más impreciso se muestra Josep Lagares, quién para denostar el comunicado alude al sentido común y a unos innominados estudios, eso sí avalados por la ciencia: "La publicación de este estudio desafía el sentido común, y también desafía numerosos estudios avalados por la ciencia que indican que no hay ninguna correlación entre cáncer y carne....". Lagares, como hombre de la industria alimentaria, no ahorra esfuerzos para refutar la mala prensa de la carne. Sin dar pistas sobre el origen de sus datos proclama que "los seguidores de la dieta mediterránea doblan el consumo recomendado de carnes procesada y, no obstante, disfrutan de las mejores esperanzas de vida". Todo ello, sin obviar las bondades de la carnes que "son una fuente de nutrientes esenciales para la vida".

Otro de los artículos de Quim Monzó, “Ahoga un mar de informaciones” (06.11.205)" rezuma escepticismo y cuestiona la credibilidad de la OMS recordando alarmas anteriores, como la de las vacas locas, que a su 
parecer, "acabaron en casi nada".

El hecho de que según el autor, las ventas de carne hayan subido, es prueba de que nadie se cree nada excepto que "de alguna cosa tenemos que morir". La banalización del comunicado mediante los dichos populares es evidente.

La recomendación más frecuente será una llamada al equilibrio y la moderación en el consumo de carne: "La cosa está, una vez más, en recordar las bondades de la dieta mediterránea y su invitación a comer un poquito de todo" (Cristina Jolonch, 21.10.2015); "..moderación y variedad, y la verdad es que ambas son un buen recurso ante muchos de los peligros de la alimentación" (Magda Carlas, 31.10.2015); "El consumo moderado de carne, junto con las nuevas tecnologías debería permitir alargar la proteína, reducir el rechazo y distribuir equitativamente su consumo" (Josep Lagares, 01.11.2015). Algunos como Francesc-Marc Álvaro ponen este discurso en boca de unos "expertos" que aparecen en televisión para decir "que no hay para tanto y que sólo se trata de comer con moderación y variedad", ya que, por si no había quedado claro, "Nada en exceso es bueno".

En general los artículos y columnas siguen la línea reflejada en los editoriales de cada uno de sus periódicos. En El Correo los textos mayoritarios se posicionan a favor de la OMS. El Mundo publica textos plagados de argumentos desdramatizadores, resaltando sobre todo los fallos en la comunicación de la OMS, y sembrando el escepticismo sobre su credibilidad, El País apela también a los fallos en la comunicación y utiliza la estrategia del absurdo para deslegitimarlo. Por último, La Vanguardia, al igual que en sus editoriales es muy crítico con el comunicado y es el que más incide en las pérdidas económicas como consecuencia principal.

Por supuesto, todos ellos publican alguna columna o editorial que se sitúa en las antípodas de su línea editorial, pero su presencia es absolutamente minoritaria.

\subsection{Cartas al director}

Durante el periodo analizado se publicaron 6 cartas al director. Tanto La Vanguardia como El País insertan dos y El Mundo y El Correo una.

De estas seis piezas, sólo dos se muestran favorables al comunicado de la OMS. Así, en El Correo, en la única misiva que publica, titulada "Demasiada carne" (28.10.2015), Martín Sagrera (Madrid) ofrece argumentos económicos, solidarios, sanitarios y medioambientales para limitar el consumo de carne. "Una dieta a base de carne cuesta el triple. También agrava la desnutrición de una gran parte de la humanidad. De ahí que por mi salud, por mi economía, por mi planeta, y por solidaridad con los demás y también con los animales....", dice el autor, no sin antes alabar a la OMS por haber roto el "muro de intereses" que intentaban disimular las verdaderas consecuencias del consumo de carne.

Isabel Vázquez Salinas escribe en El País -"El aviso de la OMS sobre la carne"- (02.11.2015) otra de las cartas que se muestra favorable a la organización y entra a comentar los efectos perniciosos de la carne. Este planteamiento es menos habitual, tanto en el apartado de cartas al director, como en el de Opinión, en general. La autora señala concretamente a los insecticidas vertidos en los pastos y los piensos como causantes de los efectos cancerígenos y nos hace responsables a todos ya que "...incorporamos a nuestro organismo todos los venenos acumulados en toda la cadena".

Sin embargo, las consecuencias, entendidas como pérdidas económicas por parte del sector alimentario también son esgrimidas en las misivas. Beatriz García Corral en su carta, "SOS alimentación" (El País, 28.10.2015), sintetiza muy bien el sentir general del sector ganadero: "Esta información puede causar mucho daño al sector ganadero y a la industria agroalimentaria porque el miedo es libre y muchas personas temerosas de contraer una enfermedad tan grave pueden dejar de consumir estas carnes con el consiguiente perjuicio".

Beatriz García pública también el mismo día en El Mundo, una carta en defensa del sector cárnico, donde con un ligero matiz repite el mismo argumento. "El sector cárnico rechaza estos términos y pide precaución" (El Mundo, 28.10.2015) y "El sector cárnico, como no podía ser de otro modo, rechaza estos términos y pide 
precaución" (El País, 28.10.2015). Tal despliegue epistolar por parte de una misma firma resulta llamativo.

En estas cartas también se esgrimen todo tipo de argumentos para desacreditar o al menos contrarrestar la gravedad del comunicado. José María Lorente, por ejemplo, en La Vanguardia (29.10.2015) resta veracidad al informe basándose en su propia experiencia: "No recuerdo ningún alimento que los científicos hayan puesto en cuestión y del que no se haya dudado sobre si era perjudicial para el corazón, o si incrementaba las posibilidades de contraer cáncer..".

Y la mayoría coincide con los columnistas al recomendar equilibrio y moderación. Jaume Font ( La Vanguardia, 28.10.2015): "Si seguimos la dieta mediterránea y la consumimos con variedad no tendremos que preocuparnos por estas alerta", ....". En el mismo diario José María Lorente concluye: "come poca cantidad y mucha variedad, pero teniendo presente siempre que vivir mata" (La Vanguardia, 29.10.2015).

De nuevo, las coincidencias con las línea de los editoriales de cada medio resultan notables.

\section{RESULTADOS}

Del análisis del discurso y de contenido se desprende que el marco interpretativo prioritario dentro de los textos analizados fue crítico. El estudio de las diferentes funciones del encuadre revela las diferencias, relevancia e incluso ausencia de alguna de ellas dependiendo del enfoque privilegiado en cada caso.

A continuación se pasa a detallar cuáles fueron los marcos interpretativos utilizados, las funciones de encuadre a las que respondieron en cada caso, así como los argumentos utilizados para sostenerlos.

\subsection{Marcos interpretativos}

El análisis revela que hubo dos enfoques: uno favorable al comunicado remitido por la OMS y otro crítico. Sin embargo, esta bipolaridad no debe ocultar la presencia en uno u otro marco de algunos argumentos compartidos por ambos, dando lugar eventualmente a marcos híbridos.

La mayoría de los textos fueron críticos y su propósito estuvo dirigido a atenuar la alarma inicial. De los 30 contenidos estudiados, sólo 6 fueron claramente positivos. En términos porcentuales un $18 \%$ de las piezas de opinión se decantaron por valorar el comunicado de la OMS en términos elogiosos. El reparto por géneros fue muy equitativo: 2 editoriales, 1 artículo de opinión, 1 columna, y 2 cartas al director.

Tabla 3. Marcos interpretativos

\begin{tabular}{|l|c|c|c|c|c|}
\hline Marcos interpretativos & El Correo & El Mundo & El País & La Vanguardia & Total \\
\hline A favor: alarma justificada & 3 & 1 & 2 & 0 & $\mathbf{6}$ \\
\hline Crítico: alarma injustificada & 2 & 6 & 6 & 10 & $\mathbf{2 4}$ \\
\hline Total & $\mathbf{5}$ & $\mathbf{7}$ & $\mathbf{8}$ & $\mathbf{1 0}$ & $\mathbf{3 0}$ \\
\hline
\end{tabular}

Fuente: Elaboración propia

El diario que más apostó por un enfoque favorable fue El Correo, que priorizó a través de su único editorial, una carta y una columna los aspectos positivos del comunicado.

En El País, su segundo editorial (01.11.2015) puso el acento en las causas y responsabilizó directamente a las industrias cárnicas. En el mismo diario una carta al director ahondó en las propiedades cancerígenas de la carne. Aunque estos dos textos participaban de un enfoque positivo, lo cierto es que también incorporaban algunos argumentos más propios del encuadre crítico. A esta hibridación fue más proclive El País, entremezclando en sus contenidos argumentos de uno u otro enfoque.

En El Mundo, Álvaro Colomer, con un titular expresivo y contundente "Chuletón de estupidez", rebatió los argumentos más populistas que denostaban el comunicado de la OMS, rompiendo una lanza a su favor.

Sólo La Vanguardia, se mantuvo al margen de este marco interpretativo decantándose firmemente por un 
marco crítico.

5.1.1. Marco interpretativo: Alarma justificada

Tabla 4. Marco interpretativo: Alarma justificada

\begin{tabular}{|c|c|c|}
\hline Dispositivos de encuadre & Argumentación & ARGUMENTOS \\
\hline \multirow{5}{*}{ IDENTIDAD/DIAGNÓSTICO } & \multirow{5}{*}{ A favor } & Rigor /método científico \\
\hline & & Universalidad del mensaje \\
\hline & & Evidencias estadísticas \\
\hline & & Dictamen inapelable \\
\hline & & Riesgo/Alarma \\
\hline \multirow[b]{2}{*}{ CAUSAS } & \multirow[t]{2}{*}{ Cadena alimentaria } & $\begin{array}{l}\text { Mala alimentación del } \\
\text { ganado }\end{array}$ \\
\hline & & Ingredientes perjudiciales \\
\hline \multirow[t]{2}{*}{ CONSECUENCIAS } & Sanitarias & Produce cáncer \\
\hline & Económicas & Dieta más cara \\
\hline RESPONSABILIDAD & \multicolumn{2}{|c|}{ Industria cárnica } \\
\hline \multirow{3}{*}{ SOLUCIONES/RECOMENDACIONES } & Político/Judiciales & Legislar/ concienciar \\
\hline & \multirow[t]{2}{*}{ Nutricionales } & $\begin{array}{l}\text { Dieta } \\
\text { equilibrada/moderación }\end{array}$ \\
\hline & & $\begin{array}{l}\text { Reducir la ingesta de } \\
\text { carne }\end{array}$ \\
\hline
\end{tabular}

Fuente: Elaboración propia

Este marco interpretativo utilizó las funciones del encuadre para presentar argumentos que respaldasen la construcción de un contexto favorable al comunicado y su contenido.

El “Diagnóstico” se apoyó en varios presupuestos para dar validez al estudio:

-El rigor científico del comunicado avalado por el consenso en la oncología médica.

-La universalidad del mensaje.

-Evidencias estadísticas.

Las "Causas" de la carcinogenicidad de la carne curiosamente fueron exhibidas en las cartas al director, tanto de El Correo, ("Demasiada carne", 29.10.2015), como de El País ("El aviso de la OMS sobre la carne", 02.11.2015) y en el editorial del 1 de noviembre también de El País. Este último fue uno de los más contundentes a la hora de señalar las causas y destacar la responsabilidad de la industria cárnica. Llama la atención que este tipo de análisis fuese pasado por alto por la mayoría de columnistas y editorialistas.

La columna de José María Romera en El Correo (30.10.2015) haciendo gala de una gran ironía, y aunque sin adentrarse en el fondo del problema, también ratificaba el contenido del comunicado y ridiculizaba al sector cárnico.

Asimismo, las "Consecuencias" sanitarias que eran una de las conclusiones más destacadas del estudio, y por tanto imposible de obviar, sólo fueron enfatizadas en estos contenidos. Las "Consecuencias" económicas en 
este marco tienen un cariz diferente al que se presenta en el marco crítico, y aluden, en un solo texto, al excesivo gasto que supondría alimentarse a base de una dieta solo de carne.

Las "Soluciones/Recomendaciones", al igual que en algunos de los contenidos más críticos, se centraron en el equilibrio y la llamada a las instituciones políticas y legislativas para asumir medidas de control y concienciación sobre los beneficios de una alimentación saludable. Sólo El País, apostó porque la industria cárnica invirtiese en investigación biológica.

\subsubsection{Marco interpretativo: Alarma Injustificada}

Este marco interpretativo se construyó por medio de aquellos argumentos que estuvieron destinados a atenuar y suavizar el contenido del comunicado de la OMS.

Estos enunciados, que se han denominado argumentos desdramatizadores, se insertaron en las funciones de "Diagnóstico", "Consecuencias" y "Soluciones/ Recomendaciones".

En este enfoque las "Causas" que remiten directamente a la alimentación del ganado o al uso de conservantes y otros productos en la elaboración de las carnes procesadas fueron obviadas en la mayoría de los casos. El País en su editorial del 1 de noviembre hizo referencia a las mismas, utilizando un marco interpretativo híbrido. Algo parecido ocurriría con la "Responsabilidad", que sólo en casos puntuales señalaba a las instituciones políticas y legislativas como incapaces de tomar las medidas necesarias para controlar la trazabilidad de los alimentos e imponer hábitos saludables.

Así, los argumentos más reiterados giraron en torno a los siguientes ejes temáticos:

\section{Gráfico 2. Argumentos desdramatizadores}

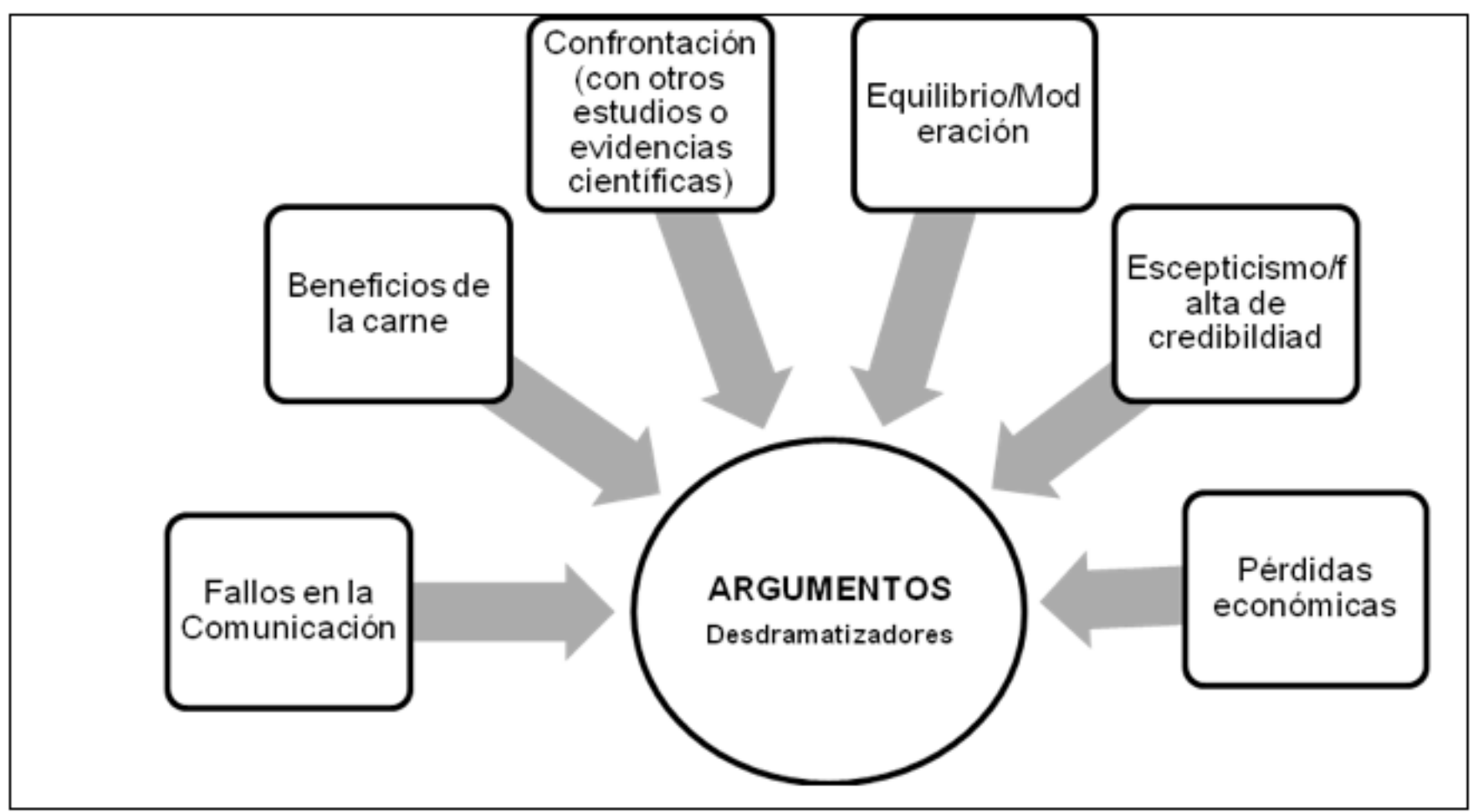

Fuente: Elaboración propia

\section{DIAGNÓSTICO}

El diagnóstico crítico, se centró tanto en deslegitimar el comunicado como en revalorizar los beneficios de la carne y los argumentos esgrimidos fueron:

-“Fallos en la comunicación de riesgos". La complejidad de una buena comunicación de riesgos da lugar a diversas críticas en torno al comunicado remitido por la OMS. Son muchos los errores que se le imputan señalando, entre otros, "ambigüedad", "inconcreción", "omisión de cifras concretas" y hablar de riesgos relativos 
y no absolutos. El diario El Mundo extendió estas críticas a los propios medios de comunicación.

-"Escepticismo/Falta de credibilidad". El desprestigio, avalado por otros expertos, o simplemente el cuestionamiento de los resultados obtenidos en base a otros informes de la misma organización en momentos anteriores fueron algunas de las armas esgrimidas para socavar la credibilidad de la OMS.

-“Confrontación (con otras evidencias y estudios científicos)". La necesidad de contrarrestar las pruebas científicas que la OMS presentaba dio lugar a una serie de argumentos que fundamentados en estudios, nominados u anónimos, contradecían o mitigaban los resultados del informe.

-"Beneficios de la carne". Los valores nutritivos, incluso culturales en forma de hábitos alimenticios, y hasta solidarios (acabar con el hambre en el mundo), son esgrimidos en este periodo.

\section{Tabla 5. Diagnóstico}

\begin{tabular}{lccccc} 
DIAGNOSTICO & El Correo & El Mundo & El País & La Vanguardia & Total \\
\hline Fallos comunicación & 1 & 3 & 4 & 4 & $\mathbf{1 2}$ \\
Escepticismo & & 2 & & 2 & $\mathbf{4}$ \\
Confrontación & & & 1 & 2 & 3 \\
Beneficios & & & 1 & 2 & 3 \\
\hline
\end{tabular}

Fuente: Elaboración propia

De todos los argumentos esgrimidos a la hora de evaluar el comunicado, los que hacían referencia a los fallos cometidos por la OMS al comunicar los riesgos para la salud fueron los más recurrentes, ya que estuvieron presentes en 12 contenidos, casi en la mitad de las piezas publicadas. Los errores en la comunicación se hicieron extensibles a los medios de comunicación, sobre todo en La Vanguardia y en El Mundo. Este último fue en este sentido el más autocrítico.

Tanto El País ("Alertas y Alarmas sobre la carne", 29.10.2015), como El Mundo ("Carnes procesadas y cáncer, un binomio mediático", 29.10.2015) hicieron de este argumento el eje central de sus editoriales.

Si exceptuamos a El Correo, que sólo en una ocasión echó mano de estos argumentos, el reparto entre los medios fue muy equitativo.

El Mundo y La Vanguardia también deslegitimaron el comunicado, insuflando la duda sobre la valía de los expertos o el rigor de sus conclusiones. El mismo objetivo se buscaba con aquellos argumentos que confrontaban la investigación de la OMS con otros estudios e informes y al que recurrieron El País y La Vanguardia en una y dos ocasiones, respectivamente.

De todas las razones esgrimidas aquellas que apelaron a los beneficios de la carne, aunque se mencionaron en tres piezas, tuvieron un papel más bien anecdótico. El País (27.10.2015) menciona su valor emocional y cultural y en La Vanguardia (01.11.2015) en uno de sus editoriales se destaca su valor proteínico.

\section{CONSECUENCIAS}

En este enfoque crítico las consecuencias sanitarias derivadas del consumo de la carne roja o elaborada son mencionadas aludiendo directamente a las conclusiones del estudio y rara vez se incide en ellas. Frente a estas, las consecuencias económicas, son mucho más relevantes, y no tratan sobre los costes económicos de una dieta fundamentalmente cárnica, sino de las pérdidas económicas que afectan al sector ganadero y la industria agroalimentaria. 
Tabla 6. Consecuencias

\section{CONSECUENCIAS \\ (Pérdidas económicas) \\ El Correo El Mundo El País La Vanguardia Total}

$\begin{array}{lllll}\text { Central } & 1 & 1 & 3 & 5 \\ \text { Complementario } & 1 & 1 & 2 \\ \text { Total } & & & 7\end{array}$

Fuente: Elaboración propia

La Vanguardia fue uno de los medios donde las consecuencias expresadas en términos de pérdidas económicas tuvieron mayor relevancia tanto en sus editoriales como en los artículos. De hecho, en su segundo editorial publicado el 1 de noviembre reconocía abiertamente el perjuicio económico que el comunicado suponía para Cataluña, donde la exportación de carne y las inversiones del sector eran realmente importantes.

En El Mundo y El País estos argumentos tuvieron un papel más secundario y complementario. Sólo en dos cartas al director, firmadas por la misma persona y publicadas el 2 de octubre el razonamiento se centraba en advertir sobre el daño provocado al sector ganadero y a la industria alimentaria.

\section{SOLUCIONES / RECOMENDACIÓN}

- Equilibrio / Moderación.

Cuando los datos expuestos por la OMS se aceptaron como evidencias científicas, los discursos se centraron en contextualizar los efectos del consumo de carne en el ámbito más local, llamar a la moderación o cantar las excelencias de la dieta mediterránea, por ejemplo. La moderación y el "sentido común" se imponen en los argumentos de esta categoría.

Hay que hacer constar que este razonamiento también estuvo presente en el marco interpretativo que justificaba la alarma de la OMS, aunque no fuese tan relevante.

Este argumento se puede hallar en todos los textos publicados en $\mathrm{El}$ Correo, en el $80 \%$ de los insertados en $\mathrm{La}$ Vanguardia, en la mitad de los contenidos de El País, 5, y en un 20\% de los recogidos por El Mundo, 2.

Este último diario es el que menos empleó este modelo de argumentación, mientras que en el resto fue una de las recomendaciones más reiteradas. Moderación, contención, equilibrio, dieta saludable y prudencia fueron algunas de las palabras clave.

\section{CONCLUSIONES}

De lo anteriormente expuesto se puede concluir lo siguiente:

- El comunicado de la OMS respecto a las consecuencias del consumo de carne roja y elaborada fue en los periódicos analizados un asunto relevante y destacado en la agenda temática. El número de editoriales (6), cartas al director (6), artículos y columnas de opinión (18), así como su prolongación en el tiempo, lo refrendan.

- De los dispositivos utilizados, así como de los argumentos empleados se desprenden dos marcos interpretativos. Uno de ellos a favor, que interpreta los hechos como una "alarma justificada", y otro de ellos crítico, del que se desprende la idea de estar ante una "alarma injustificada".

- El marco favorable fue minoritario con 6 textos, descollando el marco crítico con 24.

- El enfoque a favor del comunicado se construyó acentuando las causas, las consecuencias sanitarias y haciendo recaer la responsabilidad en la industria cárnica, sobre todo.

- En el marco crítico en general se obviaron las causas, las consecuencias sanitarias se minimizaron y se 
acentuaron las perdidas económicas del sector cárnico, diluyendo su responsabilidad.

- El enfoque crítico se valió de una serie de argumentos, que se han denominado desdramatizadores, para atenuar, minimizar y suavizar el contenido del comunicado y sus posibles consecuencias, sobre todo económicas. Los "Fallos en la comunicación" fue uno de los más utilizados, junto al "Escepticismo", "Confrontación" y "Beneficios".

- Las consecuencias económicas en términos de pérdidas en el sector y la industria cárnica estuvieron presentes en todos los medios, excepto El Correo, siendo un argumento central en al menos dos artículos y un editorial de La Vanguardia.

- El "Equilibrio / Moderación" fue un argumento presente en el 63\% (19) de los textos globales, aunque cualitativamente tuvo una relevancia especial en aquellos contenidos que responden al enfoque crítico, donde se destacó especialmente.

- Si atendemos a los enfoques prioritarios en cada medio, hay que decir que El Correo fue el diario que empleó un marco más positivo y favorable al comunicado. El Mundo fue muy crítico, poniendo el énfasis en los fallos de la comunicación en momentos de crisis. El País, fue el más equilibrado y su marco interpretativo puede ser calificado de híbrido pues utilizó argumentos tanto de uno como de otro signo. Este último fue uno de los pocos diarios cuya línea editorial remarcó las causas y las responsabilidades del sector ganadero.

- La Vanguardia optó por un marco interpretativo crítico sin fisuras y explotó los argumentos de pérdidas económicas como su razonamiento estrella.

- De análisis se desprende que hay una sintonía clara entre la línea editorial de los medios y los artículos, columnas y cartas al director. Sólo excepcionalmente se publican textos que contradicen el marco interpretativo mayoritario en cada periódico.

- Aunque en algunos textos se hace referencia a la responsabilidad de los medios de comunicación a la hora de amplificar o atenuar la alarma, lo cierto es que muy pocos artículos o editoriales recurren a fuentes expertas o estudios contrastados para validar sus planteamientos. La escasez de análisis profundos sobre las causas y responsabilidades plantea serias dudas sobre el grado de objetividad de los medios en este tema y su posible sujeción ocasional a interés externos.

El estudio de los cambios, si los hubiera, en la percepción del riesgo que tenían los lectores de los diarios analizados sería de gran interés para comprobar su influencia real en esta materia. Dejamos esta hipótesis para futuros estudios, aunque creemos haber demostrado al menos la responsabilidad de los medios de comunicación en la percepción social del riesgo a través de la elección consciente por parte de los mismos de diferentes marcos interpretativos.

* Este artículo es producto del proyecto de investigación CSO2014-54385-R, del Programa Estatal de Investigación, Desarrollo e Innovación Orientada a los Retos de la Sociedad, financiado por el Ministerio de Economía y Competividad

\section{REFERENCIAS BIBLIOGRÁFICAS Y HEMEROGRÁFICAS}

ABRIL, N (1999): Periodismo de opinión. Madrid: Síntesis.

ALCÍBAR, M.: "Propuesta pragmático-discursiva para analizar artículos editoriales: modelos y estrategias". Estudios sobre el Mensaje Periodístico, vol. 21, (2015), nº 1, pp. 225-241.

ATANASOVA, D. y KOTEYKO, N.: "Obesity frames and counter-frames in British and German online newspapers”. Health, (2016), pp. 1-20, doi: 10.1177/1363459316649764 [Consulta: 10 mayo 2016]

BECK, U. (2002): La sociedad del riesgo. Hacia una nueva modernidad. Barcelona: Paidós. 
http://www.who.int/mediacentre/news/releases/2015/cancer-red-meat/es/ [Consulta: 20 abril 2016]

CUVARDIC, D.: "Los marcos interpretativos textuales: herramienta metodológica para el análisis del discurso periodístico”. Ciencias Sociales, 96, (2002), pp. 83-98.

Declaración de la OMS sobre los vínculos entre la carne procesada y el cáncer colorrectal. 29/102015. http://www.who.int/mediacentre/news/statements/2015/processed-meat-cancer/es/. [Consulta: 20 abril 2016]

EILDERS, Ch. y LÜTERS, A.: "Research note: Germany at war. Competing framing Strategies in German Públic Discourse”, European Journal of Communication, vol. 15 (2000), n 3, pp. 415-428.

ENGUIX, O. y GALLARDO, B.: "El encuadre de los temas de salud: cobertura en prensa escrita del daño cerebral adquirido". Ámbitos. Revista Internacional de Comunicación, n 26, tercer trimestre (otoño, 2014). http://institucional.us.es/ambitos/2014/el-encuadre-de-los-temas-de-salud-cobertura-en-prensa-escrita-deldano-cerebral-adquirido. [Consulta: 25 abril 2016]

ENTMAN, R. M.: "Framing: Toward Clarification of a Factured Paradigm", Journal of Communication, vol. 43, (1993), n 4, pp. 51-58.

FARRÉ COMA, J.: "Comunicación de riesgo y espirales del miedo. Comunicación y Sociedad, 3, (2005), pp. 95119.

GAITÁN MOYA, J.A. y PIÑUEL RAIGÁDA, J.L. (1998): Técnicas de Investigación en Comunicación Social . Madrid: Síntesis.

GIL CALVO, E.: "Riesgo, incertidumbre y medios de comunicación”, Madrid, R evista Catalana de Seguretat Pública 16, (2006), pp. 103-114.

GONZALO, J.I. y FARRÉ, J. (2011): Teoría de la Comunicación de riesgo. Barcelona: UOC.

HOLSTI, O.R. (1969): Content analysis for the social sciences and humanities. Reading, Massachusetts: Addison-Wesley

JAEGER, CC.; RENN, O.; ROSA, EA.; WEBLER, T. (2001): Risk, Uncertainty and Rational Action. London: Earthscan.

LÖFSTEDT, R. E.: "Risk communication guidelines for Europe: a modest proposition." Journal of Risk Research, vol 13, (2010), (1), pp. 87-109.

LÓPEZ, J.A.; LUJÁN, J.L. (2000): Ciencia y política del riesgo. Madrid: Alianza.

LÓPEZ RABADÁN, P.: "Nuevas vías para el estudio del framing periodístico. La noción de estrategia de encuadre". Estudios sobre el Mensaje Periodístico. 16, (2010), pp. 235-258.

MOSTAZA, B.: “Editoriales”, en González Ruiz, N. (dir.) (1966): Enciclopedia del periodismo. Madrid: Noguer, pp. 171-197.

PAN, Z., y KOSICKI, G.M.: "Framing Analysis: An Approach to news discourse". Political Communication,10, (1993), pp. 55-75.

RUTSAERT, P. y REGAN, Á.: "The use of social media in food risk and benefit communication", Trends in food science and technology, vol. 30, (2013), n 1, pp. 84-91.

SÁDABA, T. (2007): Framing: el encuadre de las noticias. El binomio terrorismo-medios. Buenos Aires: La Crujía.

SANTAMARÍA, L y CASALS, M.J. (2000): La opinión periodística. Argumentos y géneros para la persuasión. Madrid: Fragua. 
SAURA, P. (2005): La gestión y la comunicación de crisis en el sector de alimentación y bebidas . Madrid: Universidad Pontificia Comillas.

SEMETKO, H. y VALKENBURG, P.M.: "Framing European Politics: A Content Analysis of Press and Television News," Journal of Communication, vol. 50, (2000), n² 2, pp. 93-109.

VAN DIJK, T (1997): Racismo y análisis crítico de los medios. Barcelona: Paidós Comunicación.

VAN GORP, B.: "Where is the frame? Victims and intruders in the Belgian press coverage of the asylum issue". European Journal of Communication, vol. 20, (2005), n 4, pp. 484-507.

VAN GORP, B.: “The Constructionist Approach to Framing: Bringing Culture Back In”. Journal of Comunication, 57, (2007), pp. 60-78.

\section{BREVE SEMBLANZA DE LOS AUTORES}

José Ignacio Armentia Vizuete, es catedrático de Periodismo y responsable del grupo consolidado Medialker, especializado en la investigación de contenidos de la prensa. Ha participado en la elaboración de los informes sobre la Seguridad alimentaria en la prensa vasca durante el periodo 2012-2015.

Flora Marín Murillo, es Profesora titular del departamento de Comunicación Audiovisual y Publicidad. En los últimos años ha centrado sus investigaciones en el tratamiento de la violencia de género por parte de la prensa y en la Seguridad alimentaria en los medios.

María Elena Olabarri Fernández, es catedrática de Comunicación Audiovisual y Publicidad. Sus líneas de investigación han estado centradas en el comportamiento del consumidor y en la eficacia publicitaria

Ámbitos. Revista Internacional de Comunicación, n.34, edición de otoño, 2016.

Recibido: 16/06/2016

Aprobado: 22/08/2016 